\title{
A comparison of physiological responses and rating of perceived exertion in two modes of aerobic exercise in men and women over 50 years of age
}

\author{
S Grant, K Corbett, K Todd, C Davies, T Aitchison, N Mutrie, J Byrne, E Henderson, \\ H J Dargie
}

Br J Sports Med 2002;36:276-281

See end of article for authors' affiliations

......................

Correspondence to: Dr G rant, Institute of Biomedical and Life

Sciences, University of

G lasgow, 640 akfield

Avenue, G lasgow G12 8

LT, Scotland, UK:

S.Grant@bio.gla.ac.uk

Accepted

25 February 2002

\begin{abstract}
Objectives: To compare the physiological responses and ratings of perceived exertion to aerobic dance and walking sessions completed at a self selected pace.

Methods: Six women and six men with a sample mean (SD) age of 68 (7) years completed aerobic dance and walking sessions in random order. A treadmill test was performed by each subject from which peak oxygen uptake $\left(\dot{V}_{2}\right)$ and maximum heart rates ( $\mathrm{HRmax}$ ) were determined. During the aerobic dance and walking sessions, heart rate and $\dot{V}_{\mathrm{O}_{2}}$ were measured continuously throughout. Rate of perceived exertion (RPE) was measured every three minutes throughout the session.

Results: The sample means (SD) for \%peak $\mathrm{VO}_{2}$ were 67 (17)\% for the aerobic dance sessions and 52 $(10) \%$ for the walking sessions, and the \%HRmax sample means (SD) were 74 (12)\% for the aerobic dance sessions and 60 (8)\% for walking sessions. The sample mean (SD) RPE for the aerobic dance sessions was $11(2)$, and for the walking sessions it was 10 (2).

Conclusions: \%peak $\dot{V}_{\mathrm{O}_{2}}$ \%HRmax, and RPE were significantly higher for aerobic dance than for walking. However, both the aerobic dance and walking sessions were of adequate intensity to improve aerobic fitness in most subjects. Further investigation into the relation between RPE and \%peak $\mathrm{V}_{2}$ in a field setting over representative exercise time periods would be useful.
\end{abstract}

A $\mathrm{s}$ the proportion of older adults in the population continues to grow, there is a great need to establish ways of delaying or preventing morbidity in later life and maintaining quality of life. ${ }^{1}$ Exercise has been shown to have a favourable effect on a variety of variables in older adults. ${ }^{2-4}$ Thus, older adults have been targeted as a sector of the population in need of specific encouragement to undertake physical activity. ${ }^{5}$

It is acknowledged that low physical activity and fitness levels are associated with ill health. ${ }^{6-9}$ Although there is evidence that exercise can be of benefit to health, it must be regular if these benefits are to be attained/maintained. Thus, it is important that regular physical activity is encouraged and that appropriate exercise sessions are promoted. However, the mode of exercise must be acceptable to the target population-that is, the participants must find the activity enjoyable and be able to participate regularly_ and it may be necessary to show that the exercise prescription is effective in improving fitness levels. The American College of Sports $M$ edicine $(\text { ACSM })^{10}$ emphasises that endurance training in the elderly has the potential to improve peak oxygen uptake $\left(\mathrm{VO}_{2}\right)$ and submaximal performance. The concept of "a little exercise is better than nothing" has been promoted. ${ }^{11}$

A variety of exercise possibilities exists for the ageing population. Indoor group exercise sessions are sociable but normally necessitate travel. Walking is easily accessible but may prove to be unpopular at certain times of the year. The cold of winter may be a deterrent in some locations and high summer temperatures may limit participation in other areas.

A previous study showed that there was no significant difference in adherence levels for aerobic dance sessions compared with walking sessions over a six month period. ${ }^{12}$ In this study a comparison was made of exercise heart rates with a small subsample of the aerobic dance and walking groups. The results based on exercise heart rates suggested that participants of both modes of exercise were exercising at an

\begin{tabular}{lll} 
Table 1 & Physical characteristics of subjects $(n=12)$ \\
\hline Characteristic & Sample mean & Range \\
\hline Age (years) & $68.0(7.1)$ & $54-78$ \\
Body mass $(\mathrm{kg})$ & $69.1(14.1)$ & $53-101$ \\
Peak $\dot{V}_{2}(\mathrm{ml} / \mathrm{kg} / \mathrm{min})$ & $29.6(7.0)$ & $19-45$ \\
HRmax (beats/ $\mathrm{min})$ & $173.7(18.8)$ & $136-210$ \\
\hline
\end{tabular}

$\dot{V}_{O_{2}}, 0$ xygen uptake; HRmax, maximum heart rate.

appropriate intensity (according to $\mathrm{ACSM}^{10}$ ) to promote/ maintain aerobic fitness. However, it is not clear if the exercise heart rates in this population are a true reflection of the metabolic cost of the activity expressed in oxygen consumption. The relation between metabolic cost and heart rate can be affected by the mode of exercise. For example, Parker $\& \mathrm{al}^{13}$ showed that exercise heart rates during an aerobic dance session did not reflect the metabolic cost of the exercise, eliciting unrepresentatively high heart rates for any given \%peak $\mathrm{\forall O}_{2}$. Although the intensity of aerobic dance and walking sessions has been assessed in young and middle aged subjects, ${ }^{14}{ }^{15}$ we know of no study that has compared the physiological responses to aerobic dance and walking sessions at self selected intensities in a population over 50 years of age.

It is often not possible to provide participants in exercise sessions with heart rate recorders. Participants are normally asked to monitor heart rate by palpation of the radial or

Abbreviations: $\dot{V}_{\mathrm{O}_{2}}$, oxygen uptake; HRmax, maximum heart rate; RPE, rating of perceived exertion; ACSM, American College of Sports Medicine 
Table 2 Summary of repeated measures analyses of va riance

\begin{tabular}{llll}
\hline & \multicolumn{2}{l}{ Variable } & \\
\cline { 2 - 4 } Factor & $\forall \mathrm{O}_{2}$ & $\mathrm{RPE}$ & $\mathrm{HR}$ \\
\hline Time & $<0.001$ & $<0.001$ & $<0.001$ \\
Mode & $<0.001$ & $<0.001$ & $<0.001$ \\
Sex & 0.17 & 0.56 & 0.42 \\
Sex $\times$ mode & $<0.05$ & $<0.001$ & 0.23 \\
Time $\times$ mode & 0.11 & 0.61 & 0.04 \\
Time $\times$ sex & 0.81 & 0.90 & 0.59 \\
Time $\times$ sex $\times$ mode & 0.81 & 0.99 & 0.92 \\
\hline
\end{tabular}

$\dot{V}_{0_{2}}$, O xygen uptake; RPE, rating of perceived exertion; HR, heart rate.

\begin{tabular}{|c|c|c|c|c|c|c|}
\hline \multirow[b]{2}{*}{ Time (min) } & \multicolumn{2}{|c|}{$\%$ peak $\forall \mathrm{O}_{2}$} & \multicolumn{2}{|l|}{ \%HRmax } & \multicolumn{2}{|l|}{ RPE } \\
\hline & $A D$ & Walk & $A D$ & Walk & $A D$ & Walk \\
\hline 3 & 57 & 47 & 65 & 57 & 10 & 9 \\
\hline 6 & 72 & 51 & 72 & 59 & 11 & 10 \\
\hline 9 & 71 & 52 & 74 & 60 & 11 & 10 \\
\hline 12 & 70 & 53 & 76 & 62 & 12 & 10 \\
\hline 15 & 64 & 52 & 76 & 61 & 12 & 10 \\
\hline 18 & 71 & 55 & 78 & 62 & 12 & 10 \\
\hline O verall mean (SD) & 67 (17) & $52(10)$ & $74(12)$ & $60(8)$ & $11(2)$ & $10(2)$ \\
\hline
\end{tabular}

$\dot{V}_{\mathrm{O}_{2}}, \mathrm{O}$ xygen uptake; HRmax, maximum heart rate; RPE, rating of perceived exertion; AD, a erobic dance.

carotid artery. However, this technique is often subject to considerable error and may result in subjects exercising at an inappropriate intensity. The substitution of heart rate monitoring with a rating of perceived exertion (RPE) 15 point scale (the Borg 6-20 scale ${ }^{16}$ ) could have advantages if it could be shown that the RPE scores were closely related to oxygen uptake. A good relation between RPE and oxygen uptake may allow intensity to be monitored using RPE.

Thus, it is important to determine the relative intensities of these aerobic dance and walking sessions and to assess the appropriateness of available methods to monitor intensity, namely, heart rate and RPE. The aim of this study was to compare the physiological responses (as determined by oxygen consumption, heart rate) and RPE to aerobic dance and walking sessions completed at a self selected pace.

\section{METHODS}

Twelve subjects ( 6 women), with a sample mean (SD) age of 68 (7) years, volunteered for the study. They were recruited from the 55+ exercise classes run by the Sport and Recreation Service of the University of Glasgow. All volunteers were regular exercisers- that is, they regularly carried out at least two aerobic bouts of exercise a week. The study was approved by the local ethics committee before the start, and all subjects completed a consent form and underwent medical screening before testing. Potential subjects taking drugs that could have influenced the haemodynamic responses to exercise were excluded from the study.

The following three tests were performed by each subject: a class based aerobic session ( 18 minutes); a self paced walk on an indoor track ( 18 minutes); a treadmill (peak $\forall^{O_{2}}$ ) test.

The aerobic dance and walking tests were carried out in random order to balance out any bias or familiarisation effect.

Details of the three tests are given below.

Test 1: aerobic dance session

This session was conducted in a sports hall and organised by Sport and Recreation Service of the University of Glasgow. An
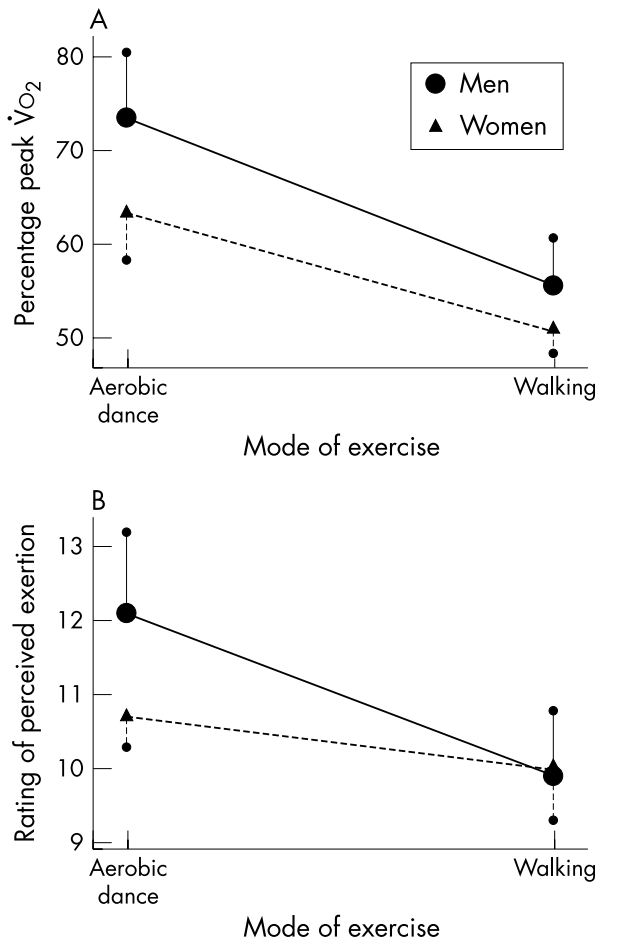

Figure 1 Plots illustrating the significant interaction of sex and mode of exercise for (A) \% peak $\mathrm{VO}_{2}$ and (B) rating of perceived exertion. The plots are of the sample means (across all six times), with vertical lines indicating one standard error of each sample mean.

instructor led the exercise session using a microphone. There were about 40 participants in the class. Subjects performed rhythmical continuous movements in time with the music. They were encouraged to regulate intensity by adjusting the 
magnitude of movement to enable them to exercise comfortably and "at their own pace". In addition, they were shown how to carry out movements keeping one foot on the floor at all times. Previous research has shown that relative intensity can be reduced by adapting movements. ${ }^{15}$

Before each test the subject's body mass was measured. Heart rate was recorded using a PE 3000 sports tester, and expired air collected by a Douglas Bag connected by a Hans Rudolph valve and Velconia tubing to a mouthpiece. An experimenter supported the Douglas bag, stopcock, and some of the Velconia tubing so that the subjects had unrestricted movement. Previous studies have used the same method of gas collection and shown that it does not restrict movement. ${ }^{15}$

A nose clip was attached to the subject's nose. The duration of gas collection was 18 minutes as this coincided with the aerobic component of the exercise session. Gas samples were of three minutes duration, and heart rate was recorded throughout, an average being collected for each three minute period. During the second minute of each three minute period, subjects were shown the Borg scale (category scale 6-20) and asked to point to the number on the scale that best described their perceived effort at that moment. Before the session began, subjects were given practice with the oxygen uptake apparatus and asked to exercise as they normally would without the apparatus.

Test 2: self paced walk

This test was conducted on an indoor track. The protocol and equipment were the same as test 1 except that the subjects were asked to walk at a "brisk but comfortable" pace for an 18 minute period. The subjects completed the self paced walk with only one experimenter who carried the Douglas bag. The variables measured were the same as for the aerobic dance session-that is, heart rate, $\forall \mathrm{O}_{2}$, and RPE. In both test 1 and test 2 , guidance on the use of the RPE scale was given before the tests.

Test 3: treadmill (peak $\forall \mathrm{O}_{2}$ ) test

This test was carried out so that the relative intensity of the two exercise modes could be evaluated and HRmax established. Tests were conducted in the exercise testing laboratory of the Western Infirmary, Glasgow. In accordance with safety criteria, a cardiologist was present at all times. Before each test, the subject's mass was measured, and a 12 lead electrocardiograph attached to allow examination of resting and exercise el ectrocardiograms.

Subjects were given a familiarisation period of walking on the treadmill ( $M$ arquette) before the test started. The STEEP protocol ${ }^{17}$ was used for the treadmill test. This test was developed so that it could accommodate a fairly wide range of aerobic fitness levels. The initial treadmill speed was 1.7 miles per hour. Intensity was then increased every minute in an exponential manner by either increasing speed or gradient. Heart rate was monitored continuously using the electrocardiograph, and gas sampling was as for tests 1 and 2 except that one minute samples were taken in the later stages of the test. Subjects were asked to continue on the treadmill for as long as possible. All subjects performed the test to volitional fatigue without incident.

RPE

Before the aerobic dance and walking session, the subjects were familiarised with the RPE scale. Instructions were based on those recommended by Pollock $\&$ al. ${ }^{18}$ Subjects were asked to rate the total amount of exertion that they felt. They were asked to concentrate on the total feeling of exertion and not to focus on any one factor.

Equipment for gas analysis

Expired air was collected using a mouthpiece, Hans Rudolph 2600 valve, tubing, and noseclip. Gases were analysed using a PK M organ $\mathrm{CO}_{2}$ analyser (type 201A) and Taylor Servomex $\mathrm{O}_{2}$ analyser (type 570A). Volume was measured using a Parkinson Cowan dry gas meter. All analysers were calibrated before each test with known gas concentrations.

\section{Statistical analysis}

Repeated measures analyses of variance were carried out separately for the three variables (heart rate, $\forall \mathrm{O}_{2}$, and RPE) with time into the test and mode of exercise (aerobic dance and walking) as "repeated measures" factors within each of the 12 subjects. A sex effect and appropriate interactions among all these factors were also included in the analyses. Standard Pearson's sample correlations were used to assess the relation between RPE and \%HRmax and then between RPE and \%peak $\mathrm{BO}_{2}$ within each subject separately and then summarised across subjects by sample medians and ranges.

\section{RESULTS}

Table 1 gives the physical characteristics and maximal values of the subjects. Table 2 provides the repeated measures analyses of variance and shows that there is a significant mode effect for all three variables-that is, $\mathrm{VO}_{2}$, heart rate, and RPE are significantly higher in the aerobic dance session than in the walking session. This mode effect is illustrated in table 3 , which gives the sample means for \%peak $\forall_{\mathrm{O}_{2}} \% \mathrm{HRmax}$, and RPE for the aerobic dance and walking sessions for each of the six three minute time periods. There is a significant time effect, with values for all three variables increasing progressively through the sessions. The repeated measures analysis of variance also shows that there are significant sex $\times$ mode interactions for both \%peak $\forall \mathrm{O}_{2}$ and RPE. This interaction indicates that there is a significantly greater difference in both \%peak $\mathrm{VO}_{2}$ and RPE between the sexes during the aerobic dance sessions than during the walking sessions ( fig 1 ).

For heart rate, there was a much less substantial-that is, compared with the mode effect-but still significant time $x$ mode interaction. This interaction indicates that the difference between the aerobic dance and walking sessions is less in the early part of the sessions compared with the later part of

Table 4 Summary statistics of within subject sample correlations between rating of perceived exertion (RPE) and percentage of heart rate maximum (\%HRmax) and between RPE and peak $\dot{V}_{\mathrm{O}_{2}}$ for a erobic dance and walking sessions

\begin{tabular}{llllll}
\hline & \multicolumn{2}{l}{ Aerobic dance sessions } & & \multicolumn{2}{l}{ Walking sessions } \\
\cline { 2 - 3 } Summary statistics & RPE and \%HRmax & RPE and \%peak $\forall \mathrm{O}_{2}$ & & RPE and \%HRmax & RPE and \%peak $\forall \mathrm{O}_{2}$ \\
\hline M inimum & -0.37 & -0.41 & -0.46 & 0 \\
Inner quartile & 0 & 0 & 0 & 0 \\
M edian & 0.23 & 0 & 0 & 0 \\
O uter quartile & 0.76 & 0.64 & 0.66 & 0.51 \\
M aximum & 0.98 & 0.96 & 0.94 & 0.90 \\
\hline
\end{tabular}


the sessions. All 12 subjects had higher \%peak $\forall \mathrm{O}_{2}$ and $\%$ HRmax at all time points in the aerobic dance session compared with the walking session. For RPE values, one subject rated the walking session harder than the aerobic dance session.

Sample (within subject) correlations were evaluated between RPE and \%HR max and then between RPE and \%peak $\mathrm{VO}_{2}$ for each of the 12 subjects separately. Table 4 gives summaries for each set of sample correlations across the 12 subjects. It is clear that, in general, there is an extremely poor, if non-existent, correlation between RPE and either \%HRmax or \%peak $\forall \mathrm{O}_{2}$, as the median (across all 12 subjects) correlations are almost all zero. The only minor exception is a possible positive but moderate correlation between RPE and $\% H R$ max where the sample median correlation reaches 0.23 . Some of the within subject correlations were zero, as these subjects recorded exactly the same RPE values at all time points on that session.

\section{DISCUSSION}

We believe that these data are representative of actual participation in these types of sessions-that is, the aerobic dance data were collected during an exercise class in a gymnasium while the walking session data were gathered on an indoor track, which was therefore not influenced by environmental factors. The ACSM ${ }^{19}$ guidelines indicate that intensity should be $50-85 \%$ of peak $\forall \mathrm{O}_{2}$ and $65-90 \%$ of $\mathrm{HRmax}$ to improve aerobic fitness. However, the guidelines cite several studies that show that people with low fitness levels can achieve a significant training effect with \%peak $\forall_{2}$ below $50 \%$. Several studies have shown that elderly subjects can improve aerobic power by exercising at a range of intensities. ${ }^{20}{ }^{21}$ Badenhop $\mathbb{A}$ $\mathrm{al}^{22}$ found that subjects with a sample mean age of 68 years who trained at $57-70 \%$ peak $\forall_{\mathrm{O}_{2}}$ increased peak $\mathrm{VO}_{2}$ by $14 \%$ over a nine week programme. Gossard et $\mathrm{al}^{23}$ showed that middle aged men improved their aerobic power while they trained at around or under $45 \%$ of peak $\forall_{\mathrm{O}_{2}}$. Thus, it seems that the sample means of $67 \%$ and $52 \%$ of peak $\forall_{2}$ for the aerobic dance and walking sessions respectively in this study could result in the maintenance or promotion of aerobic fitness in most subjects if achieved on a regular basis. A possible criticism of these sessions is the short duration time of 18 minutes. However, previous studies in a predominantly middle aged group showed an improvement in aerobic fitness with group aerobic sessions of 20 minutes two to three times a week. ${ }^{24}$ In addition, several researchers advocate that intermittent exercise bouts of fairly short duration have the potential to improve aerobic fitness and promote health benefit. ${ }^{11}$ Several studies have shown improvements in peak $\mathrm{VO}_{2}$ with short, intermittent bouts of exercise. ${ }^{25-27}$

Data from previous aerobic dance research ${ }^{14} 15$ have shown that younger subjects exercised at a similar \%peak $\mathrm{VO}_{2}$ to the subjects in this study-that is, $67 \%$ of peak $\forall_{2}$ for the present study compared with $65 \%$ of peak $\mathrm{VO}_{2}$ in the study by Grant et $\mathrm{al}^{15}$ of female university students. Spelman et $\mathrm{al}^{14}$ reported that their 35 year old subjects walked, on average, at $52 \%$ of peak $\forall \mathrm{O}_{2}$ (exactly the same as this study) at their self selected pace during a walking session. It is important to emphasise that the subjects in our study were regular exercisers and had relatively high peak $\mathrm{BO}_{2}$ levels for their age.

The use of \%HRmax to monitor intensity is more "user friendly" than \%peak $\forall_{\mathrm{O}_{2}}$. There is no consensus in the literature as to what a given \% $\mathrm{Hmax}$ is equivalent to in \%peak $\mathrm{VO}_{2}$. Swain \& $\mathrm{al}^{28}$ have questioned the equivalent values given by the ACSM ${ }^{19}$ for \%HRmax and \%peak $\forall \mathrm{O}_{2}$. However, the relation between \%HRmax and \%peak $\forall_{2}$ in this study is fairly close to that given by the ACSM 1990 guidelines ${ }^{19}$ as well as by M CArdle et al ${ }^{29}$ The sample means in this study of $74 \%$ of HRmax at $67 \%$ of peak $\mathrm{VO}_{2}$ for the aerobic dance session and $60 \%$ of $\mathrm{HRmax}$ at $52 \%$ of peak $\mathrm{VO}_{2}$ for the walking session show greater agreement-that is, the difference between the $\% \mathrm{HRmax}$ and \%peak $\forall_{\mathrm{O}_{2}}$ is much less-than the data from the Swain study, which reported a mean of $80 \%$ of $\mathrm{HRmax}$ at $67 \%$ of peak $\forall_{O_{2}}$. It is unclear why there is a closer margin between these two variables in this older population compared with the Swain study. These data suggest that, for this subject group, the monitoring of \%HRmax is probably fairly reflective of the relative intensity as measured by $\forall_{2}$. However, a further study is needed to compare the heart rate/ $\mathrm{O}_{2}$ relation in this population using a variety of modes of exercise.

Relative intensities are clearly influenced by the maximum values attained in the peak $\forall_{O_{2}}$ test. A HRmax of 210 beats/min for one of the subjects in this study prompted the question of the validity of this value. A cardiologist who examined the 12 lead electrocardiograph print out confirmed that the heart rate was in sinus rhythm and therefore the HRmax was indeed genuine.

The finding that a higher metabolic cost results in an increased RPE confirms previous research. ${ }^{30}$ However, these results are for an elderly population and show that, in a comparison of self paced exercise, the metabolic cost and RPEs are higher for aerobic dance than walking. It may be that participation in a group setting in the aerobic dance sessions resulted in some degree of peer pressure, which resulted in a higher intensity for the group session. It is to be expected that the use of music would lower RPE. ${ }^{31}{ }^{32}$ As music is integral to an aerobic dance session, it is not possible to evaluate this hypothesis in a study of this kind.

The sample mean RPE values of 11 (2) for the aerobic dance sessions and 10 (2) for the walking sessions of this study are slightly lower than would be expected for relative intensities of $67 \%$ and $52 \%$ of peak $\forall_{2}$ when compared with the guidelines of Birk and Birk. ${ }^{33}$ Birk and Birk indicate that RPE values of $12-15$ correspond in general to $58-89 \%$ of peak $\forall_{\mathrm{O}_{2}}$ respectively. Low RPE values for an aerobic dance session could be attributed to the subjects disassociating from perceptions of effort because of background music and the fact that they had to concentrate on the movements in the class. ${ }^{31-32}{ }^{34}$ However, the same is not true of the walking sessions. RPE guidelines from other sources do not concur exactly with the Birk and Birk relations. For example, the recommendations of Pollock and Wilmore, ${ }^{35}$ which are also cited in the ACSM 1990 guidelines, ${ }^{19}$ suggest that an RPE of $12-13$ corresponds to $50-74 \%$ of \%peak $\forall \mathrm{O}_{2}$, whereas Eston and Williams ${ }^{30}$ state that RPEs of $12-13$ are equivalent to a \%peak $\mathrm{VO}_{2}$ range of $60-80 \%$. They report a lower \%peak $\forall_{2}$ range for cycling at RPEs of 12-13, which indicates that mode can influence RPE. From a comparison of different modes of exercise cited in the literature, Eston and Williams ${ }^{30}$ concluded that the mode using smaller muscle groups is associated with a higher RPE. The above guidelines emphasise that it is not possible to be definitive about the RPE/\%peak $\forall_{2}$ relation.

Studies using young and middle aged subjects have shown that RPE underestimates intensity in walking sessions and aerobic dance. ${ }^{14}{ }^{15}$ The subjects in the study of Spelman $\& \mathrm{al}^{14}$ produced a mean RPE of 10.9 during a walk at $52 \%$ of peak $\forall \mathrm{O}_{2}$, which is slightly higher than the 10 in this study with exactly the same \%peak $\forall \mathrm{O}_{2}$. This finding prompted Spelman \& al to conclude that their subjects had underestimated the exercise intensity. Conversely, Zeni $\& \mathrm{al}^{36}$ reported that RPE levels of $13-15$ resulted in \%peak $\forall_{2}$ values within the recommended intensity range for several modes of exercise. Comparison with the relations outlined above indicates that RPE may be marginally lower in this study than expected. It is conceded that the differences are fairly small but may have some significance given that the Borg scale is 6-20. It is acknowledged that the sample size in this study is small and that it may be inappropriate to draw this inference.

Consideration should be given to the consequences of an unrepresentatively low RPE. The fact that the subjects perceived the exercise sessions to be not particularly stressful 


\section{Take home message}

Aerobic dance and walking sessions can result in the maintenance or promotion of aerobic fitness in most elderly subjects if achieved on a regular basis. Further investigation into the relation between RPE and \%peak $\dot{V}_{0}$ over representative exercise time periods would be useful.

could result in good adherence. On the other hand, if subjects rate RPE at 12 while they exercise at $70 \%$ of peak $\forall_{O_{2}}$, they may exercise at very high intensities while the RPE is "Iow", possibly triggering cardiac complications associated with very high intensity exercise.

The extent to which RPE can be substituted for \%HRmax and/or \%peak $\forall_{2}$ is limited. The fact that some of the individual subject sample correlations were zero was due to some of the subjects having exactly the same RPE values at all time points during that session. It may be unrealistic to expect a good relation between RPE and the selected physiological variables in this study, as scrutiny of the \%peak $\mathrm{\forall O}_{2}$ values shows that the subjects exercised at fairly constant intensity throughout both sessions. However, it should be noted that a median correlation of 0.89 between \%peak $\forall_{O_{2}}$ and RPE was found in an aerobic dance session of female students who had fairly stable physiological variables. ${ }^{15}$

Although these results show that \%peak $\forall \mathrm{O}_{2}, \% \mathrm{HRmax}$, and RPE are higher in the aerobic dance than in the walking sessions, it should be noted that some subjects had considerably higher $\mathrm{VO}_{2}$ and heart rate differences between modes than others. These differences are not associated with absolute values.

In this comparison of self selected exercise intensity of aerobic dance and walking, \%peak $\forall_{2}, \% H R m a x$, and RPE were significantly higher for aerobic dance than for walking. However, both the aerobic dance and walking sessions were of adequate intensity to improve aerobic fitness in most subjects. Further investigation into the relation between RPE and \%peak $\mathrm{VO}_{2}$ in a field setting over representative exercise time periods is deemed appropriate.

\section{ACKNOWLEDGEMENTS}

We acknowledge the support of the Wellcome Trust which provided a student summer vacation grant to allow this study to take place.

\section{Authors' affiliations}

S Grant, K Corbett, K Todd, N Mutrie, Institute of Biomedical and Life Sciences, University of G lasgow, G lasgow, Scotland, UK

C Davies, T Aitchison, Department of Statistics, University of G lasgow

J Byrne, E Henderson, H J Dargie, Department of Cardiology,

W estern Infirmary, G lasgow

\section{REFERENCES}

1 Buchner DM, W agner EH. Preventing frail health. Clin Geriatr M ed 1992:8:1-17.

2 Shephard RJ. The scientific basis of exercise prescribing for the very old. J Am G eriatr Soc 1990;38:62-70.

3 McMurdo MET, Burnett $L$. Randomised controlled trial of exercise in the elderly. G erontology 1992;38:292-8.

4 Puggard L, Pedersen HP, Sandager E, et al. Physical conditioning in the elderly. Scan J Med Sci Sports 1994;4:47-56

5 Bokovoy J L, Blair SN. Ageing and exercise: a health perspective. Journal of Aging and Physical Activity 1994;2:243-60.

6 Blair SN, Kohl HW, Paffenbarger RS, et al. Physical fitness and all-cause mortality. A prospective study of healthy men and women. JAMA $1989 ; 262: 2395-401$

7 Dargie HJ, Grant S. Exercise. BMJ 1991;303:910-13.
8 Blair S, Connelly J. How much physical activity should we do? The case for moderate amounts and intensities of physical activity. The Health of the $N$ ation 1994 Apr 22:1-13

9 Bouchard CR, Shephard R, Stephens T. Physical activity, fitness and health: international proceedings and consensus statement. Champaign, IL: Human Kinetics, 1994

10 American College of Sports Medicine. The recommended quantity and quality of exercise for developing and maintaining cardiorespiratory and muscular fitness in healthy adults. Med Sci Sports Exerc 1998;30:975-91

11 Pate RR, Pratt M, Blair SN, et al. Physical activity and public health. A recommendation from the $C$ entre for Disease $C$ ontrol and prevention and the American College of Sports Medicine. JAM A 1995;273:402-7.

12 Mutrie N, Blamey A, Davison R, et al. Class based and home based activities for older people. Research digest no 30, research report no 32 Edinburgh: Scottish Sports Council, 1993.

13 Parker SB, Hurley BF, Hanlon, DP, et al. Failure of target heart rate to accurately monitor intensity during aerobic dance. Med Sci Sports Exerc $1989 ; 21: 230-4$

14 Spelman CC, Pate RR, MacEra CA, et al. Self-selected exercise intensity of habitual walkers. Med Sci Sports Exerc;1993;25:1174-9.

15 Grant S, Davidson W, Aitchison T, et al. A comparison of physiological responses and rating of perceived exertion between high impact and low impact aerobic dance sessions. Eur J A ppl Physiol 1998;78:324-32.

16 Borg GAV. Psychophysical bases of perceived exertion. Med Sci Sports Exerc 1982;14:377-81.

17 Northridge DB, Grant S, Ford I, et al. N ovel exercise protocol suitable for use on a treadmill or a bicycle ergometer. Br Heart J $1990 ; 64: 313-16$

18 Pollock ML, W ilmore JH, Fox, SM. Exercise in health and disease Philadelphia: W B Saunders, 1984

19 American College of Sports Medicine. The recommended quantity and quality of exercise for developing and maintaining cardiorespiratory and muscular fitness in healthy adults. Med Sci Sports Exerc $1990 ; 22: 265-74$

20 Seals DR, Hagberg JM, Hurley BF, et al. Endurance training in older men and women. 1. Cardiovascular responses to exercise. J Appl Physiol 1984:57:1024-9.

21 Hagberg JM, M ontain SJ, Martin W H, et al. Effect of exercise training in 60 to 69 nine year-old persons with essential hypertension. Am J Cardiol $1989 ; 64: 348-53$

22 Badenhop DT, Cleary PA, Schaal SF, et al. Physiological adjustments to higher and or lower intensity exercise in elders. Med Sci Sports Exerc $1983 ; 15: 496-502$

23 Gossard D, Haskell W L, Barr Taylor C, et al. Effects of low and high intensity home based exercise training on functional capacity in healthy middle aged men. Am / Cardiol 1986;57:446-9.

24 Grant S, Aitchison T, Pettigrew AR, et al. The effects of a university fitness programme on health-related variables in previously sedentary males. Br J Sports Med 1992;26:39-44

25 Ebisu T. Splitting the distance of endurance running on cardiovascular endurance and blood lipids. Journal of Physical Education 1985;30:37-43.

26 De Busk RF, Stenerstrand M, Sheehan M, et al. Training effects of long versus short bouts of exercise in healthy subjects. A J J Cardiol 1994;65:1010-13

27 Murphy MH Hardman AE. Training effects of short and long bouts of brisk walking in sedentary women. Med Sci Sports Exerc 1998;30:152-7.

28 Swain DP, A bernathy KS, Smith CS, et al. Target heart rates for the development of cardiorespiratory fitness. Med Sci Sports Exerc 1993;26:112-16.

29 McArdle WD, Katch Fl, Katch, VL. Essentials of exercise physiology. Philadelphia: Lea and Febiger, 1994:360.

30 Eston, RG, W illiams JG. Control of exercise intensity using heart rate, perceived exertion and other non-invasive procedures. In: Eston R, Reilly $\mathrm{T}$, eds. Kinanthropometry and exercise physiology laboratory manual: tests, procedures and data. 2 nd ed. London, $\mathrm{N}$ ew York: Routledge, 2001;2:211-34

31 Potteiger, JA, Schroeder JM, G off KL. Influence of music on ratings of perceived exertion during 20 minutes of moderate intensity exercise. Percept M otor Skills 2000;91:848-54.

32 Nethery VM, Harmen PA, Taaffe DR. Sensory mediation of perceived exertion during submaximal exercise. Journal of Human M ovement Studies 1991;20:201-11

33 Birk TG, Birk CA. Use of perceived ratings of perceived exertion for exercise prescription. Sports Med 1987:15:496-502.

34 Grant S, A rmstrong G, Sutherland R, et al. Physiological and psychological responses to a university fitness session. Br J Sports Med 1993;27:162-6.

35 Pollock ML, W ilmore JH. Exercise in health and disease: evaluation and prescription for prevention and rehabilitation. 2 nd ed. Philadelphia: W B Saunders, 1990.

36 Zeni AI, Hoffman MD, Clifford PS. Energy expenditure with indoor exercise machines. JAMA 1996;275:1424-7 


\section{COMMENTARY}

This study measured the self selected exercise intensity adopted by older adults (aged 54-78 years) performing two modes of exercise (aerobic dance and walking). Aerobic dance elicited a higher intensity ( $67 \%$ of peak $\mathrm{VO}_{2}, 74 \%$ of maximum heart rate) than walking ( $52 \%$ of peak $\forall \mathrm{O}_{2}, 60 \%$ of maximum heart rate). The authors concluded that both forms of exercise were of sufficient intensity to improve aerobic fitness based on the fact that the American College of Sports M edicine recommends a minimum intensity of $50 \%$ of peak $\mathrm{WO}_{2}$. The study findings are relatively novel because there are few data on the physiological responses to self selected exercise in older people. Identifying acceptable and effective forms of exercise for such people is important not only from the point of view of reducing disease risk but also because such exercise will help them to maintain their functional capacity and thus their ability for independent living. ${ }^{1}$ Ultimately the result is a better quality of life for those who remain active into old age. ${ }^{2}$

D Stensel,

Department of Physical Education, Sports Science and Recreation M anagement, Loughborough University, Leicestershire LE11 3TU, UK; D.J.Stensel@lboro.ac.uk

\section{REFEREN CES}

1 Huang Y, M acera CA, Blair SN, et al. Physical fitness, physical activity, and functional limitation in adults aged 40 and older. Med Sci Sports Exerc 1998;30:1430-5

2 Spirduso WW, Cronin DL. Exercise dose-response effects on quality of life and independent living in older adults. Med Sci Sports Exerc $2001 ; 33: 5598-608$.

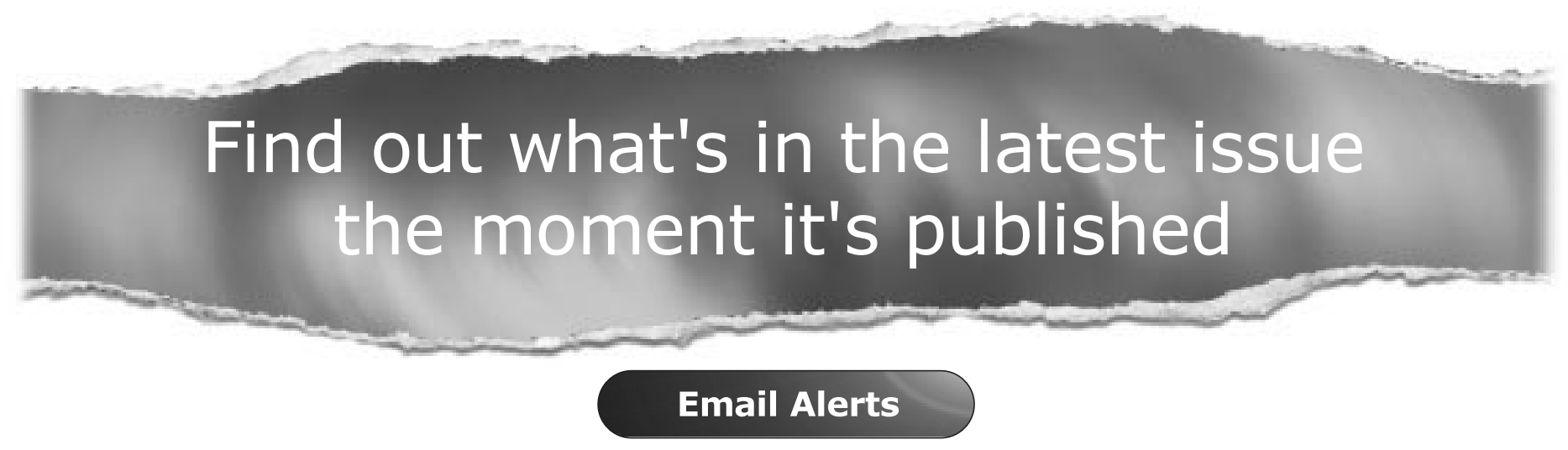

Sign up to receive the table of contents by email every month. You can select from three alerts: Table of Contents (full), TOC Awareness (notice only); British Journal of Sports Medicine related announcements.

\section{www.bjsportmed.com}

